\title{
DEPRESSÃO E QUALIDADE DE VIDA EM PACIENTES COM FIBROMIALGIA
}

\author{
Santos AMB ${ }^{1}$, Assumpção A ${ }^{1}$, Matsutani LA ${ }^{2}$, Pereira CAB ${ }^{3}$, Lage LV ${ }^{4}$ e Marques AP ${ }^{1}$ \\ ${ }^{1}$ Departamento de Fisioterapia, Fonoaudiologia e Terapia Ocupacional da Faculdade de Medicina da Universidade de \\ São Paulo - USP, São Paulo, SP - Brasil \\ ${ }^{2}$ Departamento de Fisioterapia, Centro Universitário FIEO, Osasco, SP - Brasil \\ ${ }^{3}$ Instituto de Matemática e Estatística, USP, São Paulo, SP - Brasil \\ ${ }^{4}$ Disciplina de Reumatologia, Faculdade de Medicina, USP, São Paulo, SP - Brasil \\ Correspondência para: Amélia Pasqual Marques, Departamento de Fisioterapia, Fonoaudiologia e Terapia Ocupacional, \\ Faculdade de Medicina, Universidade de São Paulo, Rua Cipotânea, 51, Cidade Universitária, CEP 05360-160, São \\ Paulo, SP - Brasil, e-mail: pasqual@usp.br
}

Recebido: 28/11/2005 - Aceito: 07/06/2006

\begin{abstract}
RESUMO
Contexto: Fibromialgia é uma síndrome reumática caracterizada por dor musculoesquelética difusa e crônica, e sítios dolorosos específicos à palpação (tender points). Freqüentemente é associada à fadiga generalizada, distúrbios do sono, rigidez matinal, dispnéia, ansiedade, alterações no humor que podem evoluir para um quadro de depressão. Sendo assim, afeta negativamente a qualidade de vida das pessoas. No entanto, ainda não se sabe o poder de discriminação dos instrumentos de avaliação da depressão e qualidade de vida. O objetivo desse estudo foi avaliar a depressão e a qualidade de vida de pacientes com fibromialgia e avaliar o poder de discriminação de instrumentos relacionados a esses aspectos. Método: Para avaliar a qualidade de vida foram utilizados dois instrumentos: um específico, o Fibromyalgia Impact Questionnaire (FIQ) e outro genérico, o Medical Outcomes Study 36-item Short-Form Healthy Survey (SF-36); para avaliar a depressão, a Escala de Depressão de Beck (BDS). Participaram do estudo 40 mulheres sendo 20 com fibromialgia - Grupo teste e 20 saudáveis - Grupo controle. Os três protocolos foram aplicados aos indivíduos dos dois grupos em uma única sessão de avaliação. Toda a análise estatística foi realizada utilizando-se o teste "t" Student, com significância $\alpha=0,05$. Resultados: Houve diferença estatisticamente significante entre os grupos controle e teste em todos os questionários $(\mathrm{p}<0,05)$. Os três questionários têm bom poder discriminatório para diferenciar os dois grupos, porém o FIQ é o que tem maior poder de discriminação e o BDS o menor. Conclusão: O grupo de pacientes com fibromialgia tem pior qualidade de vida quando comparado com o grupo controle. Comparando os três questionários todos são eficientes não somente para avaliar a qualidade de vida mas também para avaliar a depressão. Entretanto, como o FIQ é um instrumento específico é o que melhor discrimina o grupo teste do grupo controle, fato este mostrado através dos valores estatisticamente significantes mais altos.
\end{abstract}

Palavras-chave: fibromialgia, depressão, qualidade de vida.

\section{ABSTRACT \\ Depression and Quality of Life Among Patients with Fibromyalgia}

Background: Fibromyalgia is a rheumatic syndrome characterized by widespread chronic musculoskeletal pain and specific palpable tender points. It is often associated with generalized fatigue, sleep disturbances, morning stiffness, dyspnea, anxiety and mood disturbances that may evolve into depression. Thus, such individuals' quality of life is negatively affected. However, the discriminating power of quality-of-life and depression assessment instruments is still unknown. Objective: To assess depression and quality of life among patients with fibromyalgia, and the discriminating power of such instruments. Method: Quality of life was assessed by one specific instrument, the Fibromyalgia Impact Questionnaire (FIQ), and another generic one, the Medical Outcomes Study 36-item Short-Form Health Survey (SF-36). The Beck Depression Scale (BDS) was used to assess depression. Forty women participated: 20 with fibromyalgia (test group) and 20 healthy women (control group). The three protocols were applied to all individuals in both groups in a single evaluation session. All statistical analyses were performed using Student's t-test, with $\alpha=0.05$. Results: Statistically significant differences between test and control groups were found via all questionnaires $(\mathrm{p}<0.05)$. The three questionnaires had good discriminatory power for differentiating between the two 
groups, but FIQ had the greatest and BDS least. Conclusion: Patients with fibromyalgia presented poor quality of life, compared with the control group. Comparing the three questionnaires, they were all efficient for both quality-of-life and depression assessments. However, since FIQ is a specific instrument, this gave the best discrimination between test and control groups, and this was shown through higher, statistically significant values.

Key words: fibromyalgia. depression, quality of life.

\section{INTRODUÇÃO}

Fibromialgia é uma síndrome reumática que ocorre, predominantemente, em mulheres com idade entre 40 e 55 anos, caracterizada por dor musculoesquelética difusa e crônica e sítios dolorosos específicos à palpação (tender points). Freqüentemente é associada à fadiga generalizada, distúrbios do sono, rigidez matinal, dispnéia, ansiedade e alterações de humor, que podem evoluir para um quadro de depressão $0^{1,2,3,4}$. Sendo assim, o paciente fibromiálgico apresenta dificuldade em trabalhar normalmente, interferindo negativamente no desempenho de outras atividades diárias e, conseqüentemente, na qualidade de vida ${ }^{5,6}$.

A depressão é um sintoma importante sendo apontada como um dos sintomas mais freqüentes em pacientes fibromiálgicos ${ }^{1}$. Vários estudos têm utilizado a Escala de Depressão de Beck $^{7}$ para avaliar efeitos de tratamento ${ }^{8,9,10,11}$. Este questionário consiste de 21 grupos de quatro afirmações. Em cada grupo o sujeito deve escolher uma ou mais afirmações que melhor descrevem como ele se sentiu na última semana. A pontuação máxima é de 63 pontos e os altos escores indicam níveis severos de depressão. Segundo Meyer ${ }^{12}$ um escore acima de 16 indica possível depressão. Burckhardt ${ }^{13}$ usou diferentes instrumentos para avaliar a depressão em pacientes com fibromialgia e concluiu que a Escala de Depressão de Beck era o instrumento mais sensível para avaliá-la. Gowans ${ }^{8}$ usou vários instrumentos, entre eles a Escala de Beck, para avaliar a eficácia de um programa de exercícios e concluiu que o exercício físico pode melhorar o humor e a função física em indivíduos com fibromialgia.

A avaliação da qualidade de vida através de questionários tem sido reconhecida como uma importante área do conhecimento científico no campo da saúde. Isto porque os conceitos de saúde e qualidade de vida se interpõem considerados como satisfação e bem-estar nos âmbitos físico, psíquico, socioeconômico e cultural - e a prioridade nos tratamento de quaisquer doenças ou síndromes tem sido cada vez mais, a busca pela saúde, em seu âmbito mais abrangente, e a melhora da qualidade de vida. Neste sentido o uso de questionários de qualidade de vida permite uma avaliação mais objetiva desta combinação de fatores subjetivos. Na prática clínica, eles podem identificar os âmbitos mais influenciados por determinada síndrome e avaliar a efetividade de uma intervenção e da análise de custo-utilidade do tratamento ${ }^{14}$.

Estes instrumentos podem ser específicos ou genéricos. Os instrumentos específicos são capazes de avaliar, de forma particular, determinados aspectos da qualidade de vida, próprios de uma população com uma determinada doença. Em 1991, Burckhardt et al. ${ }^{15}$ propuseram e testaram um instrumento para avaliação da qualidade de vida específico para Fibromialgia, o Fibromyalgia Impact Questionnaire (FIQ). Este questionário é específico para a fibromialgia e envolve questões relacionadas à capacidade funcional, situação profissional, distúrbios psicológicos e sintomas físicos. Em grande parte das questões o maior escore indica maior impacto da fibromialgia na qualidade de vida. Desde então tem sido utilizado em situações clínicas e de pesquisa ${ }^{8,9,13,16,17,18,19}$.

White et al. ${ }^{6}$, utilizaram o FIQ e relatam que a Fibromialgia causa um impacto negativo na qualidade de vida de pacientes em idade produtiva de trabalho. Isso porque, além da dor, os sintomas de fadiga e fraqueza subjetiva causam perda da função levando à incapacidade para o trabalho e, conseqüentemente, à queda da renda familiar refletindo na qualidade de vida dessas pessoas.

Já os instrumentos genéricos foram desenvolvidos com o objetivo de estudar a qualidade de vida de indivíduos com qualquer patologia, ou mesmo de indivíduos saudáveis. Muito utilizado, o Medical Outcomes Study 36-item Short-Form Health Survey (SF-36) $)^{20}$ é um instrumento genérico e multidimensional de avaliação da qualidade de vida que engloba oito aspectos: capacidade funcional (10 itens), aspectos físicos (4 itens), dor (2 itens), estado geral de saúde (5 itens), vitalidade (4 itens), aspectos sociais (2 itens), aspectos emocionais (3 itens), saúde mental (5 itens) e mais uma questão de avaliação comparativa entre as condições de saúde atual e de um ano atrás. Cada componente varia de zero a cem, sendo zero o pior escore e, cem o melhor. Foi validado e traduzido para o português por Ciconelli ${ }^{21}$ que concluiu que este instrumento traduzido e adaptado à cultura brasileira é de fácil e rápida administração. O SF-36 tem sido utilizado como medida genérica de avaliação da qualidade de vida em condições como artrite reumatóide e osteoartrite ${ }^{22}$, além da análise do impacto na qualidade de vida em pacientes com fibromialgia ${ }^{16}$. Além disso, já foi utilizado para avaliar a qualidade de vida após intervenções medicamentosas ${ }^{17}$ e de um programa de exercícios ${ }^{18}$.

Neste sentido, é fundamental encontrar formas efetivas de avaliação dos sintomas da fibromialgia, e do impacto na qualidade de vida, uma vez que uma avaliação correta e completa pode contribuir para propor diferentes formas de abordagem a estes pacientes.

O objetivo desse estudo foi avaliar a depressão e a qualidade de vida de pacientes com fibromialgia e identificar 
qual dos instrumentos utilizados (FIQ, SF-35 e BDS) tem maior poder de discriminação entre sujeitos com e sem fibromialgia.

\section{MATERIAIS E MÉTODOS}

\section{Sujeitos}

Participaram do estudo 40 indivíduos, divididos em dois grupos:

1. Grupo Teste (GT) com 20 pacientes com diagnóstico de fibromialgia originários do Ambulatório de Reumatologia do Hospital das Clínicas da Faculdade de Medicina da Universidade de São Paulo (HCFMUSP), classificados segundo os critérios do Colégio Americano de Reumatologia ${ }^{2}$. Todos os pacientes estavam sob acompanhamento médico, com tratamento medicamentoso individualizado, e foram encaminhados ao serviço de Fisioterapia. Foram incluídos na amostra os que preenchiam os critérios de inclusão, até que se completasse o número predefinido de 20 sujeitos.

2. Grupo Controle (GC) com 20 sujeitos não fibromiálgicos, sem diagnóstico de patologias nos sistema musculoesqueletico e neurológico ou queixas incapacitantes nestes sistemas. Estes sujeitos foram selecionados por conveniência, respeitando os critérios de inclusão.

Os sujeitos foram selecionados de acordo com os seguintes critérios:

a. Idade entre 35 e 60 anos;

b. Nível cognitivo adequado para entender os procedimentos e orientações dadas;

c. Assinar o Termo de Consentimento Pós-Informação, no momento que consente em participar do estudo. A pesquisa foi aprovada pelo comitê de ética do HCFMUSP.

Não houve perda amostral em nenhum dos grupos.

Instrumentos: A avaliação dos sujeitos foi realizada utilizando-se a ficha de avaliação da Fisioterapia no ambulatório de Fibromialgia do HCFMUSP, constando dados pessoais e anamnese e os instrumentos Fibromyalgia Impact Questionnaire (FIQ), Medical Outcomes Study 36-item Short-Form Health Survey - SF-36 e Escala de depressão de Beck (BDS).

Local: Serviço de Fisioterapia do Ambulatório de Fibromialgia do Hospital das Clínicas da Universidade de São Paulo.

Procedimento: Todos os pacientes encaminhados à Fisioterapia foram entrevistados. A avaliação foi realizada na primeira sessão, previamente a qualquer intervenção fisioterapêutica e sob acompanhamento médico. Os indivíduos foram entrevistados obtendo-se dados pessoais e clínicos. As medidas de qualidade de vida foram obtidas com a aplicação do Fibromyalgia Impact Questionnaire (FIQ) ${ }^{15}$ e do Medical Outcomes Study 36-item Short-Form Health Survey (SF$36)^{20}$, versão validada para a língua portuguesa por Ciconelli ${ }^{21}$. Os dados da depressão foram obtidos com escala de depressão de Beck (BDS) ${ }^{7}$, traduzida e validada em português por Gorenstein e Andrade ${ }^{23}$.

Dada a dificuldade de leitura dos pacientes, optou-se pela avaliação na forma de leitura conjunta realizada sempre pelo mesmo examinador. Este procedimento também foi adotado no grupo controle.

\section{Análise estatística}

Para a comparação dos dois grupos, foi usado o teste t-Student para duas amostras independentes e o teste de homogeneidade com a estatística Qui-quadrado de Pearson, com correção de Yates quando necessário. Para o estudo de associação entre os resultados dos instrumentos utilizou-se a estatística t*-Student para testar a existência de correlações.

Para uma melhor comparação e possíveis composições, realizou-se a normalização dos resultados obtidos com os três questionários, FIQ, SF-36 e BDS. O objetivo foi o de que todos os índices variassem dentro dos mesmos limites, no caso entre 0 e 10. Para o cálculo do FIQ dividiu-se a variável Capacidade Funcional por 3 para que seu máximo fosse 10 como nas outras variáveis do FIQ. A medida final do FIQ é obtida pela média dos valores obtidos pelas oito variáveis relevantes. O índice assim obtido, NFIQ, é um número no intervalo de 0 a 10. Para o SF-36, dividiu-se a média das variáveis componentes do SF por 10. Dessa forma, também o índice obtido a partir deste questionário NSF36 assume valores entre 0 e 10 . O índice, NBDS, obtido a partir do BDS corresponde ao valor original do BDS multiplicado por 10 e dividido por 63 que, conseqüentemente, também varia entre 0 e 10 .

A partir dos três índices acima descritos construiu-se um Índice de Qualidade de Vida (IQL) do qual se espera que altos valores indiquem boa qualidade de vida enquanto baixos valores indicam qualidade de vida prejudicada. Como os altos valores do FIQ e BDS indicam baixa qualidade de vida, estes compõem o denominador do índice. Por outro lado, o SF36 está na direção inversa, quanto maior melhor e, portanto, foi definido como numerador do índice. A definição do IQL, utilizando-se os índices normalizados, é dada pela seguinte equação:

$$
\mathrm{IQL}=(\mathrm{NSF}-36) \div(\mathrm{NFIQ}+\mathrm{NBDS}+1)
$$

Acrescentou-se o valor 1 no denominador para impedir uma possível divisão por zero.

Toda a análise estatística foi realizada com nível de significância de 0,05.

\section{RESULTADOS}

A análise dos dados demográficos (Tabela 1) não evidenciou diferença estatisticamente significante entre os 
grupos, mostrando que eram homogêneos em relação à idade, sexo e IMC. A exceção foi a altura, que apresentou diferença estatisticamente significante $(\mathrm{p}=0,02)$, com médias $1,56 \mathrm{~cm}$ no GC e 1,61 cm no GT, fato que, no entanto, não alterou a relação entre peso e altura do IMC. Em relação ao nível de escolaridade somente $10 \%$ do grupo teste e $45 \%$ do grupo controle tinham nível universitário. Todos os participantes eram do sexo feminino.
De forma geral, grupo teste e grupo controle são estatisticamente distintos quanto à qualidade de vida e depressão, avaliados respectivamente pelo FIQ, SF-36 e Escala de Beck.

Os resultados obtidos pela aplicação do questionário específico, FIQ, evidenciou diferença estatisticamente significante entre o grupo controle e o teste (valor $\mathrm{p}<0,05$ ), para todos os seus itens, com exceção do item faltas no trabalho. Todos os itens estão listados na Tabela 2.

Tabela 1. Dados demográficos dos participantes.

\begin{tabular}{lccc}
\hline & Grupo teste (GT) & Grupo controle (GC) & \\
& Média (D.P) & Média (D.P) & $p^{*}$ \\
\hline Idade & $47,6(7,23)$ & $49,7(5,04)$ & $>0,05$ \\
Peso (Kg) & $67,86(9,44)$ & $69,12(14,12)$ & $>0,05$ \\
Altura (m) & $1,56(0,07)$ & $1,61(0,06)$ & $<0,05$ \\
IMC (Kg/m2) & $27,87(3,93)$ & $26,66(5,33)$ & $>0,05$ \\
Nível de & & & \\
escolaridade: & & $5 \%$ & \\
$1^{\circ}$ incompleto & $40 \%$ & $10 \%$ & \\
$1^{\circ}$ completo & $5 \%$ & $5 \%$ & \\
$2^{\circ}$ incompleto & $5 \%$ & $35 \%$ & \\
$2^{\circ}$ completo & $30 \%$ & $45 \%$ & \\
universitário & $10 \%$ & $0 \%$ & \\
sem estudo & $10 \%$ & $100 \%$ & \\
Sexo feminino & $100 \%$ & & \\
\hline
\end{tabular}

* $\mathrm{p}(\mathrm{Td} \leq \mathrm{t})$ bi-caudal

Tabela 2. Dados obtidos com o "Fibromyalgia Impact Questionnaire” (FIQ).

\begin{tabular}{lccc}
\hline \multicolumn{1}{c}{ Variáveis } & Grupo Teste (GT) & Grupo Controle (GC) & GC x GT \\
& Média $(D . P)$ & Média $(D . P)$ & $p^{*}$ \\
\hline 1)Capacidade funcional & $13,30(4,95)$ & $4,00(5,73)$ & $<0,01$ \\
2) Sentir-se bem & $1,05(1,09)$ & $6,05(1,66)$ & $<0,01$ \\
3)Faltas no trabalho & $0,15(0,67)$ & $0,00(0,0)$ & 0,32 \\
4) Habilidade de trabalhar & $6,75(2,81)$ & $1,69(3,02)$ & $<0,01$ \\
5) Dor & $7,75(1,87)$ & $1,98(3,30)$ & $<0,01$ \\
6) Fadiga & $8,09(2,40)$ & $3,47(3,61)$ & $<0,01$ \\
7) Sono & $8,19(1,86)$ & $2,95(3,68)$ & $<0,01$ \\
8) Rigidez matinal & $8,22(1,53)$ & $2,28(3,66)$ & $<0,01$ \\
9)Ansiedade & $8,63(1,40)$ & $4,26(3,98)$ & $<0,01$ \\
10)Depressão & $6,48(3,08)$ & $2,58(3,00)$ & $<0,01$ \\
& & & \\
\hline * & & &
\end{tabular}

* $\mathrm{p}(\mathrm{Td} \leq \mathrm{t})$ bi-caudal 
Com relação à avaliação genérica do SF-36, os grupos apresentaram, em todos os itens, diferenças estatisticamente significantes (valor $\mathrm{p}<0,05$ ). Os resultados estão listados na Tabela 3.

Relativamente à depressão, avaliada pela escala de depressão de Beck, novamente os dois grupos apresentam diferença estatisticamente significante (valor $\mathrm{p}<0,05$ ). As médias (desvios padrão) dos grupos controle e teste foram, respectivamente, 9,5 $(6,44)$ e 17,75 $(11,23)$. tem valor 8 e do grupo controle 3 enquanto para o SF-36 os valores encontram-se entre 8 e 4 e entre 4 e 2 para o BDS.

A Figura 2 mostra o gráfico de percentis do índice da qualidade de vida (IQL) dos grupos teste e controle. Esta figura ilustra a diferença da qualidade de vida existente entre o grupo teste e o grupo controle, lembrando que valores altos indicam boa qualidade de vida. Por exemplo, no percentil $80 \%$ o grupo teste tem valor próximo de zero e o controle próximo de quatro.

Tabela 3. Dados obtidos com “M.O.S. Short-form 36-item”(SF-36).

\begin{tabular}{lccc}
\hline \multicolumn{1}{c}{ Variáveis } & Grupo Teste(GT) & Grupo Controle(GC) & \multirow{2}{*}{ GC x GT } \\
& Média (D.P) & Média $(D . P)$ & $p^{*}$ \\
\hline 1)Capacidade funcional & $36,75(22,55)$ & $81,75(20,27)$ & $<0,001$ \\
2) Aspecto físico & $10,00(27,38)$ & $75,00(38,04)$ & $<0,001$ \\
3)Dor & $30,75(15,30)$ & $68,30(27,07)$ & $<0,001$ \\
4) Estado geral de saúde & $43,10(19,97)$ & $81,85(14,99)$ & $<0,001$ \\
5) Vitalidade & $26,00(20,74)$ & $62,50(23,59)$ & $<0,001$ \\
6) Aspecto social & $51,25(34,14)$ & $80,62(23,81)$ & $<0,001$ \\
7) Aspecto emocional & $41,66(45,72)$ & $69,99(40,32)$ & $<0,05$ \\
8) Saúde mental & $47,20(28,48)$ & $69,20(21,32)$ & $<0,01$ \\
& & & \\
\hline * p (Td $\leq$ t) bi-caudal & & &
\end{tabular}

Tabela 4. Dados obtidos com o “Escala de Depressão de Beck” (BDS).

\begin{tabular}{|c|c|c|c|}
\hline & Grupo Teste (GT) & Grupo Controle (GC) & \\
\hline & Média (D.P) & Média (D.P) & $p^{*}$ \\
\hline BDS & $17,75(11,23)$ & $9,5(6,44)$ & $<0,001$ \\
\hline
\end{tabular}

\section{Poder discriminatório dos questionários}

O índice de qualidade de vida (IQL) foi construído para melhor visualizar o poder discriminatório dos instrumentos utilizados. Valores altos indicam boa qualidade de vida e baixos, qualidade de vida prejudicada.

O gráfico dos percentis da Figura 1 ilustra o poder discriminatório dos três instrumentos utilizados. Todos se mostraram eficazes para a determinação do grupo que o individuo pertence. Esta qualidade é ilustrada pelas curvas de percentis devido às respectivas diferenças visuais entre as curvas correspondentes. Fica evidente pelas curvas que o FIQ é um instrumento com maior poder discriminatório do que o SF-36 que, por sua vez, tem maior poder do que o BDS. Por exemplo, no percentil 60\% o FIQ do grupo teste

\section{DISCUSSÃO}

O impacto negativo que a fibromialgia causa na qualidade de vida de indivíduos, tem sido relatado por muitos estudos , $^{5,625}$. O FIQ é um instrumento que tem sido usado em vários estudos clínicos para avaliar a função física e o impacto da fibromialgia na qualidade de vida de pacientes ${ }^{15,19}$ e para medir a eficácia de intervenções terapêuticas ${ }^{10,13,18}$, dentre elas a fisioterapia ${ }^{8,9,11}$. Neste estudo, observa-se que o FIQ é o instrumento que melhor discrimina pacientes fibromiálgicos de indivíduos saudáveis, mostrando piores índices de qualidade de vida quando comparado com o grupo não fibromiálgico.

O SF-36 é outro questionário que avalia a qualidade de vida, porém menos específico que o FIQ. Ele a avalia de modo genérico, considerando capacidade funcional, aspecto físico, dor, estado geral de saúde, vitalidade e características sociais e emocionais além de saúde mental. Os dados obtidos com este instrumento também mostram que a qualidade de vida dos fibromiálgicos é bem inferior aos indivíduos saudáveis e os domínios mais comprometidos foram o aspecto físico, a dor, a vitalidade e a capacidade funcional, em ordem decrescente. Já os domínios menos comprometidos foram o aspecto social e a saúde mental. 
Estes resultados podem ser comparados aos obtidos em outros estudos que sugerem que indivíduos com doenças crônicas, entre elas a fibromialgia, têm pior qualidade de vida do que a população saudável ${ }^{16,17,19,25}$.
O BDS, um dos instrumentos utilizados para avaliar a depressão, já foi utilizado em vários estudos para avaliar efeitos de tratamento ${ }^{8,9,10,11,25}$. No presente estudo, embora os valores sejam estatisticamente significantes, este instrumento foi o

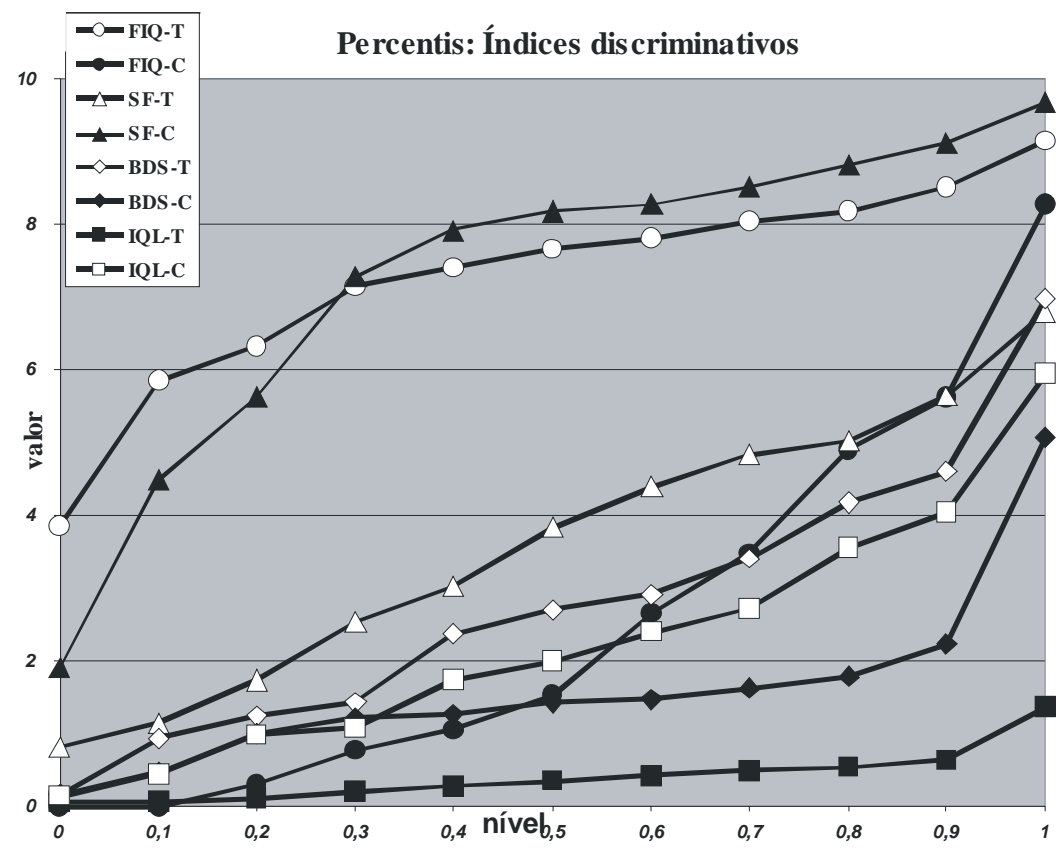

Figura 1. Representados os percentis que mostram o poder discriminatório de todos os índices. FIQ-T= FIQ teste; FIQ-C= FIQ controle SF$\mathrm{T}=\mathrm{SF}-36$ teste; $\mathrm{SF}-\mathrm{C}=\mathrm{SF}-36$ controle, BDS-T= BDS teste; BDS-C= BDS controle; IQL-T= indice de qualidade de vida Teste; IQL-C=indice de qualidade de vida Controle.

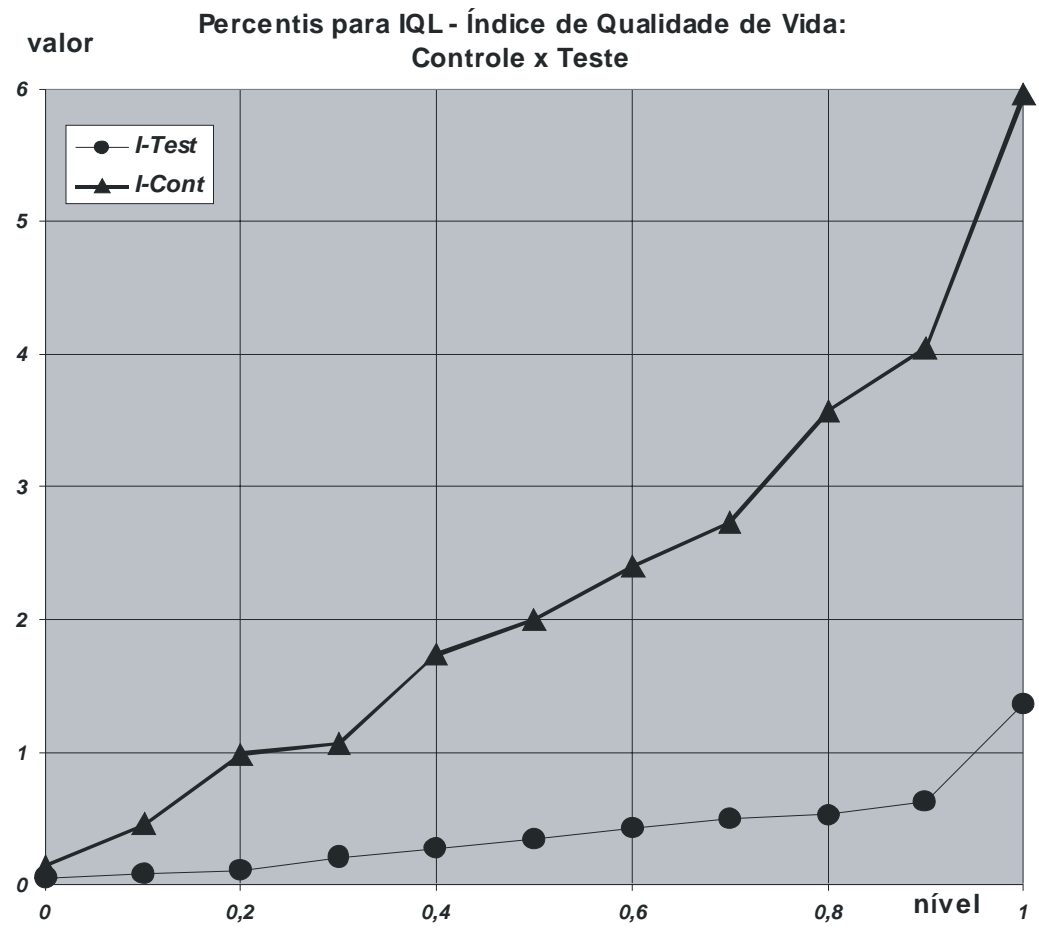

Figura 2. Gráfico de Percentis do Índice de Qualidade de Vida (IQL). Legenda: I-Test= índice teste e I-cont= Índice controle. 
que menos discriminou o grupo teste do grupo controle, provavelmente pelo fato de a depressão ser considerada um sintoma secundário na fibromialgia²

É importante observar que mesmo o grupo controle apresenta um valor diferente de zero nesta medida (Tabela 4), sugerindo que um valor esperado nulo em um estudo terapêutico não deve ser a hipótese principal quando se utiliza este instrumento como medida de depressão. Gorenstein e Andrade $^{23}$ afirmam que valores do BDS acima de 16 já indicam possível depressão. Dessa forma, é possível verificar que os pacientes com fibromialgia deste estudo apresentam este traço de depressão, aspecto este que também pode ser constatado nos resultados obtidos, de forma mais objetiva, no item depressão do FIQ com valores 6,48 e 2,58 para os grupos teste e controle respectivamente.

Como vimos, este estudo mostra que os três questionários são capazes de distinguir indivíduos com fibromialgia de saudáveis. O FIQ é o mais eficiente para caracterizar a fibromialgia, seguido pelo SF-36 e o BDS (Figura 1), o que era esperado uma vez que, este questionário foi especialmente construído para avaliar esta população. Resultados semelhantes foram relatados por Marques ${ }^{24}$.

Os resultados aqui obtidos confirmam a eficácia destes instrumentos para avaliar a qualidade de vida e depressão em indivíduos com fibromialgia, dando margem para sua utilização na prática clínica a fim de identificar as necessidades dos pacientes e avaliar a efetividade da intervenção.

\section{CONCLUSÃO}

O grupo de pacientes com fibromialgia tem pior qualidade de vida e níveis mais altos de depressão quando comparado com o grupo controle. Isto sugere que pode haver relação entre fibromialgia e depressão e que este pode ser considerado um sintoma secundário da fibromialgia.

Comparando os três questionários - FIQ, SF-36 e BDS - é possível concluir que todos são eficientes para avaliar a qualidade de vida e a depressão. Entretanto, como o FIQ é um instrumento específico, dos três é o que melhor discrimina o grupo teste do grupo controle.

\section{REFERRÊNCIAS BIBLIOGRÁFICAS}

1. Gaskin ME, Greene AF, Robinson ME, Geisser ME. Negative affect and the experience of chronic pain. Journal of Psychosomatic Research 1992; 36 (8):707-13.

2. Wolfe F, Smythe HAA, Yunus MB, Bennett AM, Bombardier CE, Goldenberg DL. The American College of Rheumatology. Criteria for the classification of fibromyalgia: Report of the Multicenter Criteria Committee. Arthritis Rheum 1990; 33(2): 160-72.

3. Weiss DJ, Kreck TE, Albert RK. Dyspnea resulting from fibromyalgia. Chest 1998; 113(1):246-9.
4. Matsutani LA, Marques AP, Carvalho CRF. Correlation between dyspnea and pain in fibromyalgic patients. Proceedings of the $9^{\text {th }}$ Annual Congress of the European Respiratory Society; 1999 October 9-13; Madrid; Spain; 1999.

5. Martinez JE, Atra E, Ferraz MB, Silva PSB. Fibromialgia: aspectos clínicos e socioeconômicos. Rev. Bras Reumatol 1992; 32: 225-30.

6. White KP, Speenchley M, Harth M, Ostbye T. Comparing selfreported function and work disability in 100 comunity cases of fibromyalgia syndrome versus controls in London, Ontario. Arthritis and Rheumatism1999; 42(1): 76-83.

7. Beck AT, Ward CH, Mendelson M, Mock J, Erbaugh J. An inventory for measuring depression. Arch Gen Psychiatry 1961; jun 4: 561-71.

8. Gowans SE, Dehueck A, Voss S, Silaj A, Abbey SE. Six-month and one-year follow up of 23 weeks of aerobic exercise for individuals with fibromyalgia. Arthritis Rheum. 2004;51(6):8908.

9. Redondo JR, Justo CM, Moraleda FV, Velayos YG, Puche JJ, Zubero JR. Long-term efficacy of therapy in patients with fibromyalgia: a physical exercise-based program and a cognitivebehavioral approach. Arthritis Rheum. 2004 Apr 15;51(2):18492.

10. Evcik D, Kizilay B, Gokçen E. The effects of balneotherapy on fibromyalgia patients. Rheumatol. Int., 2002; 22 (2): 56-9.

11. Rooks DS, Silverman CB, Kantrowitz FG. The effects of progressive strength training and aerobic exercise on muscle strength and cardiovascular fitness in women with fibromyalgia: a pilot study. Arthritis Rheum. 2002;47(1):22-8.

12. Meyer BB, Lemley KJ. Utilizing exercise to affect the symptomology of fibromyalgia: a pilot study. Med Sci Sports Exerc 2000; 32(10): 1691-7.

13. Burckhardt CS, Mannerkorpi K, Hedenberg L, Bjelle A. A randomized, controlled clinical trial of education and physical training for women with fibromyalgia. J Rheumatol 1994; 21(4):71420.

14. Carr AJ, Thompson PW, Kirwan JR. Quality of life measures. Br J Rheumatol 1996; 35(3):275-81.

15. Burckardt CS, Clark SR, Bennett RM. The Fibromyalgia Impact Questionnarie: Development end Validation. J Rheumatol 1991; 18(5): 728-33.

16. Pagano T, Matsutani LA, Ferreira EA, Marques AP, Pereira CA. Assessment of anxiety and quality of life in fibromyalgia patients. Sao Paulo Med J 2004; 122(6):252-8.

17. Bennett RM, Schein J, Kosinski MR, Hewitt DJ, Jordan DM, Rosenthal NR. Impact of fibromyalgia pain on health-related quality of life before and after treatment with tramadol/acetaminophen. Arthritis Rheum 2005; 53(4):519-27.

18. Valim V, Oliveira L, Suda A, Silva L, de Assis M, Barros Neto T, et al. Aerobic fitness effects in fibromyalgia. J Rheumatol 2003;30(5):1060-9.

19. Tikiz C, Muezzinoglu T, Pirildar T, Taskn EO, Frat A, Tuzun C. Sexual dysfunction in female subjects with fibromyalgia. J Urol 2005;174(2):620-3.

20. Ware JE, Sherborne CD. The MOS 36-item Short-Form Health Survey (SF-36). I. Conceptual framework and item selection. Med Care 1992; 30(6): 473-83. 
21. Ciconelli RM. Tradução para o português e validação do questionário genérico de avaliação da qualidade de vida "Medical Outcomes Study 36-Item Short-Form Health Survey (SF-36)" [Tese de Doutorado]. Universidade Federal de São Paulo; 1997.

22. Dias RC. Impacto de um protocolo de Fisioterapia sobre a qualidade de vida de idosos com osteoartrite de joelhos. [Tese de Doutorado]. São Paulo:Universidade Federal de São Paulo; 1999.

23. Gorenstein C, Andrade L. Validation of a portuguese version of the Beck depression inventory and state -trai anxiety inventory in brasilian subjects. Braz J Med Biol Res 1996; 29 (4): 453-7.
24. Marques AP. Qualidade de vida de indivíduos com fibromialgia: poder de discriminação dos instrumentos de avaliação. [Tese de livre docência]. São Paulo: Universidade de São Paulo;2004.

25. Schlenk EA, Erlen JA, Dunbar-Jacob J, et al. Health-related quality of life in chronic disorders: a comparison across studies using the MOS SF-36. Qual Life Res. 1998;7(1):57-65. 